\title{
La médecine immédiate
}

\section{Eberhard Wolff}

PD Dr rer. soc., rédacteur culture, histoire, société

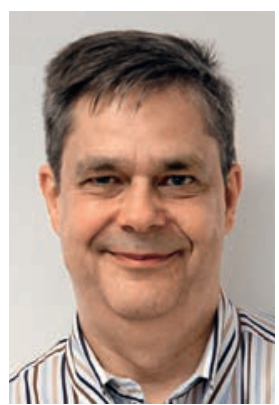

Déshabiller, sécher et réchauffer. C’est par là que commençait généralement la réanimation des noyés au XVIII ${ }^{\mathrm{e}}$ siècle. C'est du moins ce que l'on trouve dans le bestseller de Tissot, daté de 1761 [1]. Ce n'est qu'après qu'on souffle de l'air chaud dans les poumons. Non pas pour "ventiler», mais pour faire de nouveau circuler les humeurs. Tout cela était cohérent avec les concepts médicaux de l'époque.

Puis s'imposa l'idée d'un sauvetage systématique des personnes accidentées [2]. Mais chez Tissot, nous ne lisons rien sur les «mesures immédiates». L'idée selon laquelle la rapidité pouvait aussi sauver la vie a germé lentement dans la médecine. Pour autant, le tableau de sauvetage des noyés du médecin görlitzois Christian August Struve, daté de 1799 [3], ne dit pas la même chose: il estime qu'il faut sortir "promptement" les noyés de l'eau, les amener «à la hâte» dans la maison la plus proche et les déshabiller «rapidement». Les notions de rapidité et d'urgence sont manifestement relatives et variables.

Ces six derniers mois, le présent journal a plusieurs fois évoqué la question de l'urgence et de l'immédiateté dans la pratique médicale. Pour résumer, il y est écrit que les patients, mal informés et inconditionnels de la technique, ont une mentalité du "tout tout de suite», exigeant des rendez-vous et des diagnostics immédiats, et invoquant un droit à être rétablis «immédiatement" [4]. Le terme d'immédiateté couvre un aspect pressant, urgent, moral ou suspect. Bon, l'immédiateté a donc quelque chose de problématique.

Je reconnais en même temps que moi et mes enfants ont sollicité les services d'urgence plus souvent que nécessaire. Notamment le week-end. Il aurait été difficile d'obtenir un rendez-vous chez le médecin le lundi et cela aurait complètement bouleversé le programme professionnel et familial. J'ai peut-être été perçu parfois comme l'un de ces patients nerveux, que l'on trouve au même titre que des médecins impatients.

Mais les flots grossissants de patients arrivant aux urgences sont-ils l'expression de l'incivilité d'une mentalité du tout tout de suite, qui pose problème et qui s'étend? Non. Je nous perçois, moi et beaucoup d'autres patients, comme appartenant à une société qui peut, veut et peut-être doit vivre différemment d'il y a cinquante ans: pas seulement plus rapidement, mais aussi de façon plus adaptée, plus interconnectée et en étant plus sollicité. Bien sûr, tout cela va de pair avec la numérisation. Les courriels arrivent instantanément. Les réponses aussi, parfois, par exemple celle de mon rédacteur en chef, même tard le soir. Voire encore plus tard. C'est cela, plus de souplesse et d'adaptation. De moins en moins de gens s'asseyent à $19 \mathrm{~h} 30$ devant les informations. Ils vont à tout moment sur Internet chercher ce qu'ils veulent voir. Cela m'arrive aussi de plus en plus souvent.

La médecine ne fait pas exception. Avant, il fallait attendre sa radio. Aujourd'hui, elle est tout de suite sur le serveur. Nous recevons en «temps réel» les informations sur la saturation en oxygène. Mon interniste me communique mon taux de lipides sanguins peu après la prise de sang. La «médecine immédiate» est confortable, mais surtout efficace, pour mon médecin comme pour moi. Le passage du patient en cabinet normal ne fonctionne aussi que parce qu'en règle générale, tous font «immédiatement» ce qu'il y a à faire.

Ces derniers mois, le présent journal s'est aussi interrogé pour savoir si le lean management était une vision d'avenir pour l'hôpital et le cabinet [5]. L'enjeu est l'efficacité, dans la gestion du temps aussi: pas de temps morts. Dans le «lean hospital», les patients ne doivent pas non plus attendre longtemps. Nous voulons des patients satisfaits. Bon, l'immédiateté est donc une notion d'avenir.

Mais alors, quand l'immédiateté est-elle un problème, quand est-elle une solution? Cela dépend-il de qui parle, de qui a le droit de s'exprimer? Pour moi, il est tout aussi problématique de considérer l'immédiateté comme une avancée que de la dénoncer en bloc, comme un confort ou une exigence excessive. Nous avancerons si nous considérons dans un premier temps la médecine immédiate comme faisant partie de notre existence, tout comme l'intemporalité de Tissot était l'expression de son époque. Alors, nous comprendrons mieux pourquoi à l'avenir le marché du «slow med» va se développer, mieux encore, se distinguer.

Car les notions de rapidité et d'urgence sont relatives et variables. Et si Tissot n'était pas mort deux ans avant les tableaux de sauvetage de Struve, peut-être se seraitil énervé contre toute cette mentalité de l'immédiateté dans la réanimation. 